\title{
Clinical significance of novel biomarker NGAL in early diagnosis of acute renal injury
}

\author{
JINGHUA ZHANG，JIANLE HAN，JINRUI LIU，BING LIANG，XIAOBO WANG and CHANGAN WANG
}

\author{
Renal Transplant Division, Department of Nephrology, Zhengzhou No. 7 \\ People's Hospital, Zhengzhou, Henan 450017, P.R. China
}

Received May 3, 2017; Accepted September 7, 2017

DOI: $10.3892 /$ etm.2017.5150

\begin{abstract}
The present study investigated the clinical significance of the novel biomarker neutrophil gelatinase-associated lipocalin (NGAL) for the early diagnosis of acute renal injury (AKI). Thirty-eight critically ill patients with acute renal injury treated at Zhengzhou No. 7 People's Hospital between December 2015 and November 2016 served as the AKI group (observation group). At the same time, 38 critically ill patients without acute renal injury were also selected as the non-AKI group (control group). Serum NGAL and creatinine (SCr) levels were measured by enzyme-linked immunosorbent assay (ELISA) at 2, 8, 12 and $24 \mathrm{~h}$ post-operation, whereas particleenhanced turbidimetric immunoassay (PETIA) was used to quantify the levels of cysteine protease inhibitor cystatin $\mathrm{C}(\mathrm{CysC})$ in serum at 2, 8, 12 and $24 \mathrm{~h}$ post-operation. The correlations between indicators were also analyzed, with ROC curves used to evaluate the diagnostic values of NGAL, SCr and CysC in AKI. No significant differences in SCr levels were found between the two groups at different time-points after operation $(\mathrm{P}>0.05)$, but NGAL and CysC levels in the observation group were significantly higher than in the control group $(\mathrm{P}<0.05)$. Pearson correlation coefficient analysis showed NGAL and CysC were positively correlated with Scr levels. For NGAL in early diagnosis, the area under the AKI curve was 0.904 , the sensitivity was $90.2 \%$ and the specificity was $89.5 \%$; for CysC in early diagnosis, the area under the AKI curve was 0.806 , the sensitivity was $79.2 \%$ and the specificity was $78.5 \%$; for $\mathrm{SCr}$ in early diagnosis, the area under the AKI curve was 0.634 , the sensitivity was $64.2 \%$ and the specificity was $62.5 \%$. Therefore, NGAL demonstrated a satisfactory early predictive value for AKI and can be used as a biomarker for early AKI diagnosis.
\end{abstract}

Correspondence to: Dr Changan Wang, Renal Transplant Division, Department of Nephrology, Zhengzhou No. 7 People's Hospital, 17 Jingnanwu Road, Zhengzhou, Henan 450017, P.R. China E-mail: drchanganwang@163.com

Key words: acute renal injury, neutrophil gelatinase-associated lipocalin, serum creatinine, cysteine protease inhibitor cystatin $\mathrm{C}$

\section{Introduction}

Acute renal injury (AKI), also known as acute renal failure, refers to the clinical syndrome of a sudden decline in renal function during the first 3 months of a disease course (1). AKI can be caused by a variety of factors, with clinical manifestations including fluid and electrolyte balance and acid and alkaline balance disorders as well as azotemia (2). AKI incidence in critically ill patients is high (up to 30-50\%), and AKI severity is positively correlated with patient mortality, with AKI is one of the leading causes of death in critically ill patients (3). AKI can increase the burden of disease and the treatment costs of critically ill patients. At present, the pathophysiological mechanism of AKI is not yet fully understood, so improving treatment methods is a challenge. Therefore, AKI has become one of the most difficult medical problems present throughout the world $(4,5) . \mathrm{SCr}$ is often used as a diagnostic index for AKI, but SCr levels are affected by many factors (blood volume, muscle mass and protein intake), leading to its uncertain association with acute renal cell injury. As such, SCr cannot be used to accurately and timely reflect the occurrence of AKI (6). Early detection and timely treatment is the key for the treatment of AKI, so the identification of novel biomarkers of AKI for early diagnosis is particularly important. As a member of lipocalin superfamily, NGAL was considered to be one of the ideal and important biomarkers in the diagnosis of AKI (7). CysC is a member of the cysteine protease inhibitor superfamily and is frequently used as a marker for the diagnosis of AKI. In this study, we examined and analyzed NGAL levels in critically ill patients after surgery. The present study provided a theoretical basis for the early diagnosis of AKI.

\section{Materials and methods}

General information. Thirty-eight critically ill patients with acute renal injury treated at Zhengzhou No. 7 People's Hospital between December 2015 and November 2016 were selected to serve as AKI group (observation group). At the same time, 38 critically ill patients without acute renal injury were selected as the non-AKI group (control group). Inclusion criteria were as follows: i) critically ill patients with surgical treatment; ii) ICU stay $\geq 24 \mathrm{~h}$; iii) patients provided informed consent. Exclusion criteria were as follows: i) patients had 
Table I. General patient information.

\begin{tabular}{lcrr}
\hline Items & Observation group $(\mathrm{n}=38)$ & Control group (n=38) & $\mathrm{t} / \chi^{2}$ \\
\hline Sex (male/female) & $21 / 17$ & $20 / 18$ & 0.046 \\
Age range (years) & $40-75$ & $35-75$ & 0.829 \\
Average age (years) & $48.76 \pm 7.48$ & $49.07 \pm 7.86$ & 0.176 \\
MAP (mmHg) & $96.87 \pm 12.37$ & $97.35 \pm 11.58$ & 0.175 \\
WBC (10 $/$ l) & $16.06 \pm 5.93$ & $15.84 \pm 5.74$ & 0.164 \\
Serum BUN (mmol/n) & $29.03 \pm 5.83$ & $28.14 \pm 5.35$ & 0.693 \\
Type of damage (n, $\%)$ & & 0.869 \\
Acute tubular necrosis & $15(39.47)$ & $16(42.11)$ & 0.490 \\
Acute glomerular injury & $14(36.84)$ & $15(39.47)$ & 0.047 \\
Acute interstitial nephritis & $9(23.68)$ & $7(18.42)$ & 0.053 \\
\hline
\end{tabular}

Table II. NGAL level comparison (ng/ml).

\begin{tabular}{|c|c|c|c|c|c|}
\hline Group & Cases & $2 \mathrm{~h}$ & $8 \mathrm{~h}$ & $12 \mathrm{~h}$ & $24 \mathrm{~h}$ \\
\hline Observation & 38 & $81.78 \pm 3.48$ & $83.93 \pm 3.23$ & $85.29 \pm 3.48$ & $89.29 \pm 3.48$ \\
\hline Control & 38 & $23.04 \pm 3.98$ & $21.05 \pm 3.32$ & $18.06 \pm 3.59$ & $17.64 \pm 3.59$ \\
\hline t-value & & 68.941 & 83.683 & 82.889 & 88.339 \\
\hline P-value & & $<0.001$ & $<0.001$ & $<0.001$ & $<0.001$ \\
\hline
\end{tabular}

received nephrotoxic drugs within the week prior to selection; ii) patients had received conventional dialysis before admission; iii) patients with heart failure and malignant tumors. There were no significant differences in general information parameters between the two groups $(\mathrm{P}>0.05$; Table I). The study was approved by the Ethics Committee of Zhengzhou No. 7 People's Hospital.

\section{Experimental methods}

Experimental equipment and reagents. Main equipment consisted of a microplate reader (Jiangsu Potebio Biotechnology Co., Ltd., Jiangsu, China), automatic biochemical instrument (Precise, Beijing, China), centrifuge (Beijing Guangan Medical Equipment Factory), pipettes (Dragon Laboratory Instruments Ltd., Beijing, China), EP tubes and centrifuge tubes (Haimen Innovative Experimental Equipment Factory, Jiangsu, China). Experimental reagents consisted of NGAL ELISA kits (R\&D Systems, Inc., Minneapolis, MN, USA), CysC kits (Beijing Strong Biotechnologies, Inc., Beijing, China) and SCr kits (BioSino, Beijing, China).

Specimen collection. Blood samples were extracted from the radial artery of all patients at 2, 8, 12 and $24 \mathrm{~h}$ post-operation. The blood samples were centrifuged for $5 \mathrm{~min}$, and the supernatant was transferred into EP tubes and kept at $-80^{\circ} \mathrm{C}$ for storage.

Indicator detection. NGAL and Scr levels were detected using ELISA according to the manufacturer's instructions. In brief: i) blood stored at $-80^{\circ} \mathrm{C}$ was thawed at room temperature $\left(20^{\circ} \mathrm{C}\right)$; ii) samples and standards were diluted (dilution ratio 1:5) and transferred to the plate; iii) samples were incubated in the plate for $30 \mathrm{~min}$ at $37^{\circ} \mathrm{C}$ and then washed with phosphate-buffered saline (PBS) 5 times for $15 \mathrm{sec}$ for each time; iv) enzymeconjugated reagent $(50 \mu \mathrm{l})$ was added and incubated at $37^{\circ} \mathrm{C}$ for $30 \mathrm{~min}$, followed by four 15 -sec washes; v) color developer $\mathrm{A}$ and $\mathrm{B}$ were added and the samples were incubated at room temperature in the dark for $15 \mathrm{~min}$. The termination solution was then added, and the OD value at $450 \mathrm{~nm}$ was measured using a microplate reader within $15 \mathrm{~min}$. These readings were used to calculate NGAL and Scr levels.

CysC levels were detected using PETIA, under the principle that serum CysC can bind to latex particles coated with goat anti-human CysC polyclonal antibodies (dilution 1:500; cat. no. PD-RM-0006-M0001; Biomart, Wuhan, China), resulting in increased turbidity at $600 \mathrm{~nm}$ proportional to CysC levels. The main parameters of the assay were as follows: main wavelength, $600 \mathrm{~nm}$; reaction methods, point end assay; reaction direction, positive; temperature, $37^{\circ} \mathrm{C}$. The difference in OD value of the calibration solution (OD value at 5 min later - OD value at 1 min later) was calculated, and an absorbance-concentration curve for the calibration solution was established. The difference in sample OD value was then calculated and CysC level was calculated according to the absorbance-concentration curve.

Evaluation criteria. Serum NGAL and Scr levels were measured by ELISA at 2, 8, 12 and 24 h post-operation and serum CysC levels were measured by PETIA at the same time-points.

Statistical analysis. Data were processed using the SPSS 19.0 (SPSS, Inc., Chicago, IL, USA) software. Measurement 
Table III. SCr level comparison $(\mu \mathrm{mol} / \mathrm{l})$.

\begin{tabular}{lccccc}
\hline Group & Cases & $2 \mathrm{~h}$ & $8 \mathrm{~h}$ & $12 \mathrm{~h}$ & $24 \mathrm{~h}$ \\
\hline Observation & 38 & $72.18 \pm 3.54$ & $70.84 \pm 3.28$ & $73.87 \pm 3.25$ & $78.78 \pm 3.74$ \\
Control & 38 & $72.05 \pm 3.72$ & $71.03 \pm 3.62$ & $74.25 \pm 3.17$ & $79.04 \pm 3.69$ \\
t-value & & 0.156 & 0.240 & 0.516 & 0.305 \\
P-value & 0.876 & 0.811 & 0.607 & 0.761 \\
\hline
\end{tabular}

Table IV. CysC level comparison (mg/l).

\begin{tabular}{|c|c|c|c|c|c|}
\hline Group & Cases & $2 \mathrm{~h}$ & $8 \mathrm{~h}$ & $12 \mathrm{~h}$ & $24 \mathrm{~h}$ \\
\hline Observation & 38 & $4.08 \pm 0.43$ & $4.23 \pm 0.28$ & $4.39 \pm 0.38$ & $4.59 \pm 0.47$ \\
\hline Control & 38 & $2.83 \pm 0.78$ & $2.57 \pm 0.37$ & $1.73 \pm 0.49$ & $1.03 \pm 0.52$ \\
\hline t-value & & 8.651 & 22.054 & 26.444 & 31.309 \\
\hline P-value & & $<0.001$ & $<0.001$ & $<0.001$ & $<0.001$ \\
\hline
\end{tabular}

Table V. Correlation between NGAL and CysC levels and $\mathrm{SCr}$ level.

\begin{tabular}{lcc}
\hline Indication & $\mathrm{r}$ & P-value \\
\hline NGAL & 0.518 & 0.013 \\
CysC & 0.501 & 0.027 \\
\hline
\end{tabular}

data were expressed as mean \pm standard deviation (false) and comparisons between groups were performed using the t-test. Measurement data were expressed by ratio and comparison between the groups were performed using $\chi^{2}$ test. Pearson correlation coefficient analysis was carried out for correlation analysis. Diagnostic value was analyzed by ROC curve. $\mathrm{P}<0.05$ was considered to be statistically significant.

\section{Results}

Serum NGAL levels. The serum NGAL level in the observation group was significantly higher than in the control group at 2, 8, 12 and $24 \mathrm{~h}$ after operation $(\mathrm{P}<0.05$; Table II $)$.

Serum SCr levels. No significant difference in SCr level was found between observation and control groups at 2, 8, 12 and $24 \mathrm{~h}$ after operation $(\mathrm{P}<0.05$; Table III).

Serum CysC levels. The serum CysC level in the observation group was significantly higher than in the control group at 2,8, 12 and $24 \mathrm{~h}$ after operation $(\mathrm{P}<0.05$; Table IV).

Correlation analysis between NGAL, CysC and Scr. Pearson correlation coefficient analysis showed that NGAL and CysC levels were positively correlated with $\mathrm{SCr}$ levels $(\mathrm{P}<0.05$; Table V and Figs. 1 and 2).

Comparison of NGAL, CysC and SCr for the early diagnosis of AKI. The potential of NGAL, CysC and $\mathrm{SCr}$ for use as an

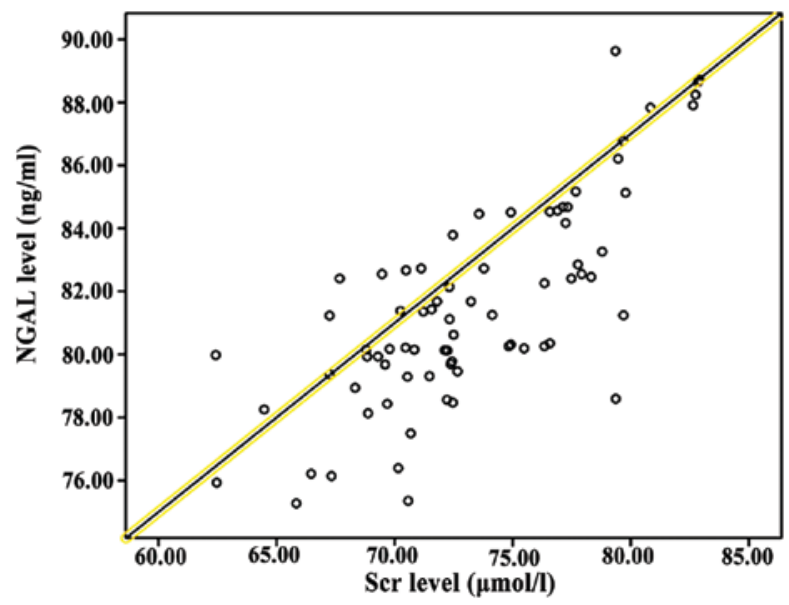

Figure 1. Correlation between NGAL and SCr levels. Pearson correlation coefficient analysis showed that NGAL levels were positively correlated with SCr levels $(\mathrm{P}<0.05)$.

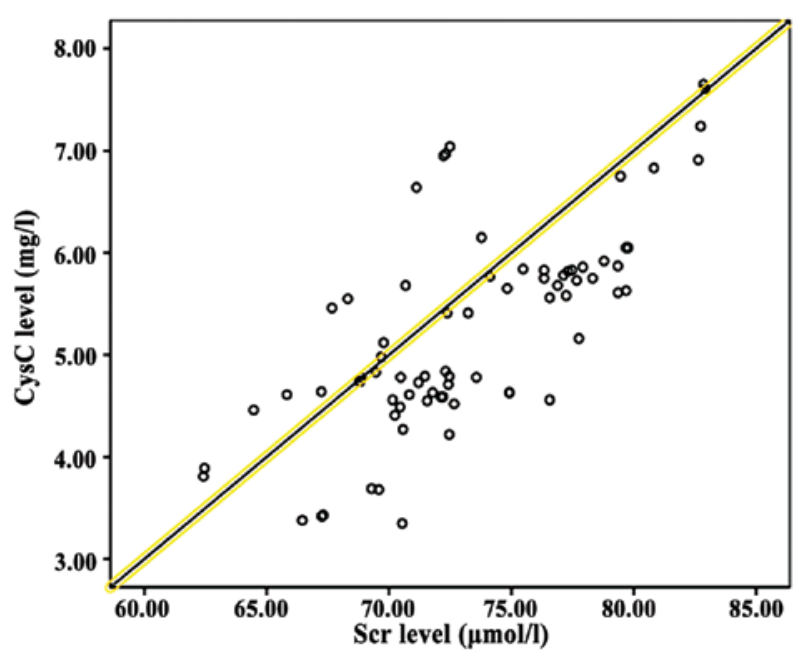

Figure 2. Correlation between NGAL and CysC levels. Pearson correlation coefficient analysis showed that NGAL levels were positively correlated with CysC levels $(\mathrm{P}<0.05)$. 


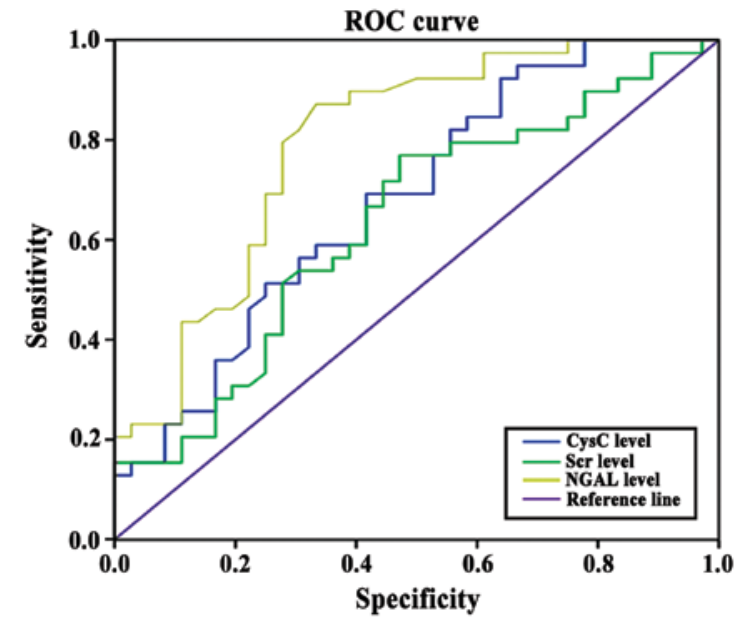

Figure 3. Comparison of NGAL, CysC and Scr for early diagnosis of AKI. The area below the AKI curve of NGAL for early diagnosis of AKI was larger than that of $\mathrm{CysC}$, and the area below the $\mathrm{AKI}$ curve of $\mathrm{CysC}$ for early diagnosis of AKI was larger than that of SCr.

early diagnostic marker of AKI was evaluated. For NGAL, the area under the AKI curve was 0.904 , the sensitivity was $90.2 \%$ and the specificity was $89.5 \%$; for CysC, the area under the AKI curve was 0.806 , the sensitivity was $79.2 \%$ and the specificity was $78.5 \%$; for Scr, the area under the AKI curve was 0.634 , the sensitivity was $64.2 \%$ and the specificity was $62.5 \%$ (Fig. 3).

\section{Discussion}

AKI is a new concept proposed by the International Renal Disease and Emergency Medicine community to replace the term 'acute renal failure'. Infectious diseases are the main causes of AKI, and other causes may include cardiogenic shock, hypovolemia, septic shock and major surgery. AKI is common for critically ill patients in the ICU. The mortality rate of AKI is high and AKI is also an independent risk factor for death in critically ill patients (8). The renal function of AKI patients can be significantly reduced within a short time, and the resultant inability to excrete metabolites reduces the stability of the internal environment. The diagnostic criteria used to identify AKI are: sudden changes in renal structure or function within $48 \mathrm{~h}$ identified by imaging, or hematuria during routine examination found within 3 months, with an increase in SCr content greater than $26.4 \mu \mathrm{mol} / 1$ or $50 \%$ of the base value; urine output below $0.5 \mathrm{ml} /(\mathrm{kg} \cdot \mathrm{h})$ for more than $6 \mathrm{~h}$, but not including patients with dehydration and obstructive nephropathy (9). Compared with the term 'acute renal failure', the term AKI covers a wider range, including renal damage from slight changes in renal function to final renal function loss. AKI can accurately reflect the developmental nature of nephropathy (10). Although the incidence of AKI is high, the awareness rate is generally low. Early symptoms of AKI are not typical, and this, coupled with the fact that diagnoses are affected by a variety of factors, results in low diagnostic sensitivity. This in turn delays diagnosis and increases mortality in critically ill patients (11). Most AKI cases are reversible, and early diagnosis and treatment can prevent the development of renal failure. Therefore, diag- nosis and treatment are keys in reducing the mortality rate in critically ill patients with AKI (12).

Urine output and changes in SCr levels are usually used to reflect changes in glomerular filtration rate and renal function decline in order to provide a basis for the diagnosis of AKI. Renal tubular damage can destroy the renal hypertonic environment and reduce the ability to concentrate urine, resulting in non-oliguric nephropathy (13). In addition, urine output, which is affected by diuretics and bladder capacity, cannot reflect renal function in real-time. Therefore, for the diagnosis of AKI, urine output and SCr levels cannot properly reflect renal injury. This misses the optimal time-point for treatment and increases patient pain and systemic economic burden (14). The results of this study showed that there was no significant difference in serum SCr level between the two groups at 2, 8, 12 and $24 \mathrm{~h}$ after operation $(\mathrm{P}>0.05)$. This was because serum $\mathrm{SCr}$ level is affected by nutritional status, muscle metabolism, and preoperative medication. Renal compensatory and reserve capacity is strong under normal conditions. SCr levels can only be increased slightly when the glomerular filtration rate is reduced by $50 \%$ or more. In addition, $\mathrm{SCr}$ accumulation takes time, and SCr levels cannot reach a stable state within a short period (6). Therefore, $\mathrm{SCr}$ cannot be used as a marker to accurately reflect short-term changes in renal function.

NGAL is a member of lipocalin superfamily with a small molecular weight of only $25 \mathrm{kDa}(15)$. NGAL was first found in human neutrophils. NGAL is very stable, so it can be easily detected in serum. Under normal conditions, low NGAL expression levels can be detected in a variety of tissues (lung, kidney, large intestine and stomach). High expression levels of NGAL can be induced by cell apoptosis after epithelial cell damage (16). The results of this study showed that serum NGAL levels in the observation group were significantly higher than in the control group at 2, 8, 12 and $24 \mathrm{~h}$ after operation $(\mathrm{P}<0.05)$. A possible mechanism for this phenomenon may be that various factors stimulated renal tubular epithelial cells, leading to increased NGAL expression. NGAL can be absorbed by renal epithelial cells to regulate the expression of apoptosis-related proteins, which in turn promotes cell maturation and induces granulocyte apoptosis (16).

CysC, a member of the cysteine protease inhibitor superfamily, is a type of non-glycosylated alkaline protein secreted by karyocytes. CysC can be synthesized at a very stable rate and released into the circulation. After complete glomerular filtration, CysC will be uptaken and degraded by nearby tubule epithelial cells. Therefore, serum CysC levels can precisely reflect the glomerular filtration rate. As such, CysC can be an ideal marker for the diagnosis of AKI (17-19). Under normal conditions, CysC levels are low. However, CysC levels can significantly increase after renal tubular injury (20). The results of this study showed that the serum CysC levels in the observation group were significantly higher than in the control group at 2, 8, 12,0 and $24 \mathrm{~h}$ after operation $(\mathrm{P}<0.05)$, indicating that internal environment of the patients was altered after AKI.

Relevant studies have confirmed that increased NGAL and CysC levels appeared 24 to $48 \mathrm{~h}$ prior to $\mathrm{SCr}$ level increases in the diagnosis of AKI (21). In the present study, we found that for NGAL in early diagnosis, the area under the AKI curve was 0.904 , the sensitivity was $90.2 \%$ and the specificity was 
89.5\%; for CysC in early diagnosis, the area under the AKI curve was 0.806 , the sensitivity was $79.2 \%$ and the specificity was $78.5 \%$; for $\mathrm{SCr}$ in early diagnosis, the area under the AKI curve was 0.634 , the sensitivity was $64.2 \%$ and the specificity was $62.5 \%$. Our findings were consistent with previous studies. In addition, the sensitivity and specificity of NGAL were higher than those of $\mathrm{CysC}$ and $\mathrm{SCr}$ for the detection of AKI. The results also showed that the levels of NGAL and CysC were positively correlated with $\mathrm{SCr}$ levels, indicating that the increased expression of NGAL and CysC were consistent with the upregulation of $\mathrm{SCr}$ expression.

In summary, evaluating NGAL levels in critically ill patients may improve the early detection of AKI and facilitate early treatment and improve prognosis.

\section{References}

1. Teles F, de Mendonça Uchôa JV, Mirelli Barreto Mendonça D and Falcão Pedrosa Costa A: Acute kidney injury in leptospirosis: The Kidney Disease Improving Global Outcomes (KDIGO) criteria and mortality. Clin Nephrol 86: 303-309, 2016.

2. Brown JR, Robb JF and Malenka DJ: Abstract 1056: Does 'safe' dosing of iodinated contrast prevent contrast-induced acute kidney injury. Cardiology 131: 249, 2015.

3. Hoste EA, Bagshaw SM, Bellomo R, Cely CM, Colman R, Cruz DN, Edipidis K, Forni LG, Gomersall CD, Govil D, et al: Epidemiology of acute kidney injury in critically ill patients: The multinational AKI-EPI study. Intensive Care Med 41: 1411-1423, 2015.

4. Wang N, Jiang L, Zhu B, Wen Y and Xi XM; Beijing Acute Kidney Injury Trial (BAKIT) Workgroup: Fluid balance and mortality in critically ill patients with acute kidney injury: A multicenter prospective epidemiological study. Crit Care 19: 371, 2015.

5. Cuartero M, Betbesé A, Sabater J, Ballús J and Ordóñez J: Urinary TIMP2 and IGFBP7 as early biomarkers of acute kidney injury in septic and nonseptic critically ill patients. Crit Care 19 (Suppl 1): 191-201, 2015.

6. Lagos-Arevalo P, Palijan A, Vertullo L, Devarajan P, Bennett MR, Sabbisetti V, Bonventre JV, Ma Q, Gottesman RD and Zappitelli M: Cystatin $\mathrm{C}$ in acute kidney injury diagnosis: Early biomarker or alternative to serum creatinine? Pediatr Nephrol 30: 665-676, 2015.

7. Veighey K and MacAllister R: Clinical applications of remote ischaemic preconditioning in native and transplant acute kidney injury. Pediatr Nephrol 30: 1749-1759, 2015.
8. Ostermann M, Dickie H and Barrett NA: Renal replacement therapy in critically ill patients with acute kidney injury - when to start. Nephrol Dial Transplant 27: 2242-2248, 2012.

9. Bagshaw SM, Zappitelli M and Chawla LS: Novel biomarkers of AKI: The challenges of progress 'Amid the noise and the haste' Nephrol Dial Transplant 28: 235-238, 2013.

10. Young P, Bailey M, Beasley R, Henderson S, Mackle D, McArthur C, McGuinness S, Mehrtens J, Myburgh J, Psirides A, et al; SPLIT Investigators; ANZICS CTG: Effect of a buffered crystalloid solution vs saline on acute kidney injury among patients in the intensive care unit: The SPLIT Randomized Clinical Trial. JAMA 314: 1701-1710, 2015.

11. Siew ED and Davenport A: The growth of acute kidney injury: A rising tide or just closer attention to detail? Kidney Int 87: 46-61, 2015 .

12. Karvellas CJ, Durand F and Nadim MK: Acute kidney injury in Cirrhosis. Crit Care Clin 31: 737-750, 2015.

13. Siew ED and Matheny ME: Choice of reference Serum creatinine in defining acute kidney injury. Nephron 131: 107-112, 2015.

14. Grynberg K, Polkinghorne KR, Ford S, Stenning F, Lew TE, Barrett JA and Summers SA: Early serum creatinine accurately predicts acute kidney injury post cardiac surgery. BMC Nephrol 18: 93, 2017.

15. de Geus HR, Betjes MG, Schaick R and Groeneveld JA: Plasma NGAL similarly predicts acute kidney injury in sepsis and nonsepsis. Biomarkers Med 7: 415-421, 2013.

16. Devarajan P: NGAL for the detection of acute kidney injury in the emergency room. Biomarkers Med 8: 217-219, 2014.

17. Yim H, Kym D, Seo DK, Yoon J, Yang HT, Lee J, Cho YS, Hur J, Chun W and Han SW: Serum cystatin C and microalbuminuria in burn patients with acute kidney injury. Eur J Clin Invest 45: 594-600, 2015.

18. Bongiovanni C, Magrini L, Salerno G, Gori CS, Cardelli P, Hur M, Buggi M and Di Somma S: Serum cystatin C for the diagnosis of acute kidney injury in patients admitted in the emergency department. Dis Markers 2015: 416059, 2015.

19. Volpon LC, Sugo EK and Carlotti AP: Diagnostic and prognostic value of serum cystatin $C$ in critically ill children with acute kidney injury. Pediatr Crit Care Med 16: e125-e131, 2015.

20. Gaygısız Ü, Aydoğdu M, Badoğlu M, Boyacı N, Güllü Z and Gürsel G: Can admission serum cystatin $\mathrm{C}$ level be an early marker subclinical acute kidney injury in critical care patients? Scand J Clin Lab Invest 76: 143-150, 2016.

21. Ghonemy TA and Amro GM: Plasma neutrophil gelatinaseassociated lipocalin (NGAL) and plasma cystatin C (CysC) as biomarker of acute kidney injury after cardiac surgery. Saudi J Kidney Dis Transpl 25: 582-588, 2014. 